\title{
PALEO
}

Revue d'archéologie préhistorique

$20 \mid 2008$

Spécial table ronde ( $2^{\mathrm{e}}$ partie) : Le Gravettien : entités régionales d'une paléoculture européenne, Les Eyzies, juillet 2004

\section{Le Gravettien de l'abri Pataud. Bilan et perspectives}

The Gravettian of the Abri Pataud. Results and prospects

\section{Roland Nespoulet}

\section{(2) OpenEdition}

\section{Journals}

Édition électronique

URL : http://journals.openedition.org/paleo/1620

DOI : $10.4000 /$ paleo. 1620

ISSN : 2101-0420

Éditeur

SAMRA

Édition imprimée

Date de publication : 1 décembre 2008

Pagination : 373-380

ISSN : 1145-3370

Référence électronique

Roland Nespoulet, «Le Gravettien de l'abri Pataud. Bilan et perspectives », PALEO [En ligne], 20 | 2008, mis en ligne le 12 mai 2011, consulté le 07 juillet 2020. URL : http://journals.openedition.org/paleo/ 1620 ; DOI : https://doi.org/10.4000/paleo.1620

\section{(ब) $\odot \Theta$}

PALEO est mis à disposition selon les termes de la licence Creative Commons Attribution - Pas d'Utilisation Commerciale - Pas de Modification 4.0 International. 


\title{
LE GRAVETTIEN DE L'ABRI PATAUD BILAN ET PERSPECTIVES
}

\author{
Roland NESPOULET ${ }^{(1)}$
}

\begin{abstract}
Résumé : Dès les premiers sondages du site, en 1953, l'importance des dépôts attribuables au Gravettien s'est imposée comme l'un des apports fondamentaux de l'archéostratigraphie de l'Abri Pataud au Paléolithique supérieur européen. Après un rappel historique et méthodologique sur les fouilles de Hallam L. Movius, la présentation de quelques données quantitatives permettra de mieux cerner à quelle série archéologique on fait référence aujourd'hui lorsque l'on évoque la collection gravettienne de l'Abri Pataud, riche de plus d'un million d'objets.
\end{abstract}

Mots-clés : Stratigraphie, typologie, collection de référence.

Abstract: The Gravettian of the Abri Pataud. Results and prospects. From the first tests excavations on the site in 1953, it was obvious that the importance of the gravettian's deposits is one of the most fundamental contributions of the Abri Pataud's archeostratigraphy to the European Upper Palaeolithic. We will begin by an historical and methodological reminder on the Hallam $\mathrm{L}$. Movius excavations, and then some quantitative data's will be presented with the aim to define as well as possible the Abri Pataud Gravettian collection, which represents more than 1 millions artefacts.

Keywords: Stratigraphy, typology, reference collection/assemblage.

Le projet de fouilles à l'Abri Pataud, gisement connu depuis la fin du $\mathrm{XIX}^{\text {e }}$ siècle, a été initié dans les années cinquante par Hallam L. Movius. En 1953, le talus et l'avant de l'abri firent l'objet de deux sondages. La stratigraphie relevée et la quantité de vestiges découverts confirmèrent alors l'intérêt du choix initial de mener, sous l'ancienne grange Pataud, des fouilles de grande ampleur. Débutées en 1958, les fouilles de Hallam L. Movius s'arrêtèrent en 1964.

Les niveaux gravettiens furent explorés en 1958 (niveau 2 et 3), 1959 (fin du niveau 3, niveaux 4 et 5), 1960 (fin du niveau 4, niveau 5) et 1961 (niveau 5). En 1963, deux extensions furent réalisées, l'une dans le niveau $2\left(20 \mathrm{~m}^{2}\right)$ et l'autre dans le niveau $4\left(7 \mathrm{~m}^{2}\right)$. Différentes méthodes de fouilles furent employées en 1958, en 1959, puis durant les années suivantes. A cette remarque s'ajoute le fait que les superficies fouillées, l'épaisseur des dépôts et la densité du matériel archéologique sont très variables d'un niveau gravettien à l'autre.

\section{Limites des études actuelles: rappel sur les méthodes de fouilles employées}

En 1958, dès les premières semaines de fouilles, une fois le sol de l'ancienne grange Pataud nettoyé, la principale difficulté rencontrée par l'équipe de fouille fut le dégagement des imposants blocs issus de l'ultime effondrement de l'abri. Une autre difficulté, comme cela a été clairement exprimé par Hallam L. Movius, fut de relever une stratigraphie claire, détaillée et fiable du premier niveau archéologique important rencontré, le niveau 2 , d'où proviennent la quasi-totalité des restes humains découverts à l'Abri Pataud (Movius 1977).

Pour tenter de remédier aux problèmes de lecture stratigraphique rencontrés dans le niveau 2, Hallam L. Movius décida de fouiller le niveau 3, sous-jacent, en laissant des banquettes témoins entre chaque carré. II abandonna ensuite cette technique : en contrepartie de la relative sécurité qu'elle apportait au point de vue stratigraphique,

(1) UMR 7194 du CNRS, Département de Préhistoire du Muséum national d'histoire naturelle, 1, rue René Panhard, 75013 Paris - nespoulr@mnhn.fr 
elle rendait impossible le décapage simultané toute la surface de fouille (ibid.). Pour le niveau 4, puis pour le niveau 5 (ainsi que pour les niveaux aurignaciens, jusqu'au substratum rocheux) une banquette centrale de 4 mètre de large fut dégagée par " surface » associée à deux tranchées latérales de contrôle stratigraphique de un mètre de large.

Un carroyage fut utilisé pour réaliser les plans et coordonner les objets. II est important de rappeler qu'une sélection, opérée sur la fouille, ne retint, pour les coordonner, que l'outillage lithique et osseux, et les objets importants diagnostiqués comme tels en place (parure, objets d'art, restes humains...). On peut ainsi estimer que sur plus d'1,5 millions d'objets effectivement extraits de la fouille, 40000 environ furent coordonnés, le reste des objets étant rassemblé par subdivisions stratigraphiques et planimétriques. Les documents scientifiques associés à la collection, constitués par les coupes et plans originaux, les carnets de numérotation d'objets, les carnets de terrain et les fiches-objets, sont d'un intérêt primordial pour localiser et replacer les objets archéologiques dans leur contexte initial.

II n'en reste pas moins vrai, et il faut insister sur ce point, que la qualité de la fouille et du travail d'inventaire fut remarquable. C'est elle, aujourd'hui, qui autorise la reprise de l'étude des collections, à l'Abri Pataud même, où les collections sont conservées.

\section{De la complexité stratigraphique à l'interprétation cul- turelle de la séquence gravettienne}

Sur les 14 niveaux principaux de la séquence stratigraphique désormais classique de l'Abri Pataud, 4 sont attribués au Gravettien. Ils prennent place dans la moitié supérieure du remplissage. La plus ancienne occupation gravettienne (niveau 5) correspond au moment de l'extension maximum de l'abri-sous-roche et la plus récente (niveau 2) est juste antérieure au dernier effondrement de la voûte. L'archéostratigraphie proposée par Hallam L. Movius, simple dans ses grandes lignes, qui fait correspondre chaque niveau à un stade du Gravettien (Périgordien IV à VII), séparée par un « éboulis » archéologiquement stérile, est bien sûr plus complexe dans la réalité, comme cela avait été remarqué à la fouille. Plusieurs exemples illustrent le type de problèmes rencontrés. Ils sont à chaque fois liés à l'interprétation stratigraphique et culturelle des niveaux.

Le niveau 5

La fin de l'Aurignacien et le début du Gravettien sont marqués, à l'Abri Pataud, par plusieurs problèmes stratigraphiques qui se traduisent par des mélanges de pièces. Le niveau 6 , qui contient moins de 400 outils, est attribué typologiquement à l'Aurignacien évolué (Brooks 1979, 1995 ; Chiotti 1999). Dans le niveau 5, situé juste au-dessus, et dont l'épaisseur atteint presque $1 \mathrm{~m}$ par endroits, des pièces aurignaciennes ont été retrouvées. Sans revenir en détail sur la présence de ces pièces, présentée par ailleurs par les fouilleurs eux-mêmes, on peut rappeler que le niveau 5 a fait l'objet d'interprétations détaillées (Bricker
1973, 1995 ; Movius 1977) que l'on peut résumer comme suit. Devant l'impossibilité d'établir des raccords stratigraphiques entre la partie avant (5:Front) $)^{2}$ et la partie arrière du dépôt (5:Rear), qui apparaissaient de toute évidence typologiquement (donc culturellement) homogènes, il fut très difficile de proposer un modèle interprétatif de ce niveau. $Y$ avait-il eu synchronie ou non des occupations des deux parties avant et arrière ? Laquelle des deux était la plus ancienne ? Ces questions apparaissent aujourd'hui encore comme étant cruciales, car elles renvoient à la caractérisation du plus ancien Gravettien du sud-ouest de la France. Pour Hallam L. Movius et Harvey M. Bricker, la réponse fut d'abord stratigraphique, puis typologique. Ils expliquèrent ainsi l'absence du niveau 6 (Aurignacien) dans la partie arrière de l'abri, par un remaniement volontaire des nouveaux arrivants gravettiens qui, en surcreusant profondément leur aire d'habitat, avaient fait disparaître l'occupation précédente. De plus, toujours selon les mêmes auteurs, le même phénomène s'est probablement reproduit ultérieurement, au point d'oblitérer les premières occupations gravettiennes du niveau 5 dans la partie arrière de l'abri. Suivant cette interprétation, la base du niveau dans partie avant de l'abri résulte du remaniement et du déblaiement de la partie arrière. Et dans tous les cas de figure, l'aire d'activité principale semble toujours avoir été située en arrière de l'abri, donc détruite par les « aménagements " successifs des différentes phases de l'occupation. Ainsi, seule l'ultime aire d'activité de la partie arrière subsiste et, selon Hallam L. Movius et Harvey M. Bricker, toute la séquence de la partie arrière est contemporaine de la partie avant supérieure.

Cette interprétation a pour conséquence de considérer que seule la partie avant basale conserve les vestiges des plus anciennes occupations. Elle est étayée par des arguments typologiques, plus précisément par la prise en compte statistique de la variation de six séries d'« attributs » des pointes de La Gravette (Bricker 1973, 1995). Mais, comme le fait remarquer Harvey M. Bricker, il apparaît que les méthodes de fouilles et d'étude du matériel utilisées, pourtant d'une qualité remarquable pour l'époque, ne permirent pas d'apporter les preuves directes de cette interprétation (Bricker 1995). En particulier, les décapages, les remontages et les données technologiques font défaut pour proposer une interprétation des aires d'activités, en place ou remaniées.

\section{Remarques sur les datations radiocarbone du niveau 5}

Le remplissage de l'abri Pataud a fait l'objet de 57 datations par la méthode du radiocarbone (dont 16 par procédé AMS). (Bricker et Mellars 1987 ; Movius 1971, 1975 ; Vogel et Waterbolk 1994). Nous ne reviendrons ici que sur les 16 dates obtenues pour le niveau 5 (dont 2 par procédé AMS). Cinq dates ont été obtenues pour la partie arrière (5:Rear Lower et 5:Rear Upper) et 11 dates ont été obtenues pour la partie avant (5:Front Lower et 5:Front Middle). Par contre, la subdivision 5:Front Upper n'a pas donné de dates). (tabl. 1, fig. 1). La répartition des dates du niveau 5 ne suit pas la logique de l'interprétation archéologique. Les

(2) La terminologie d'origine, en anglais, a été conservée. Elle apparaît en italique dans le texte. 


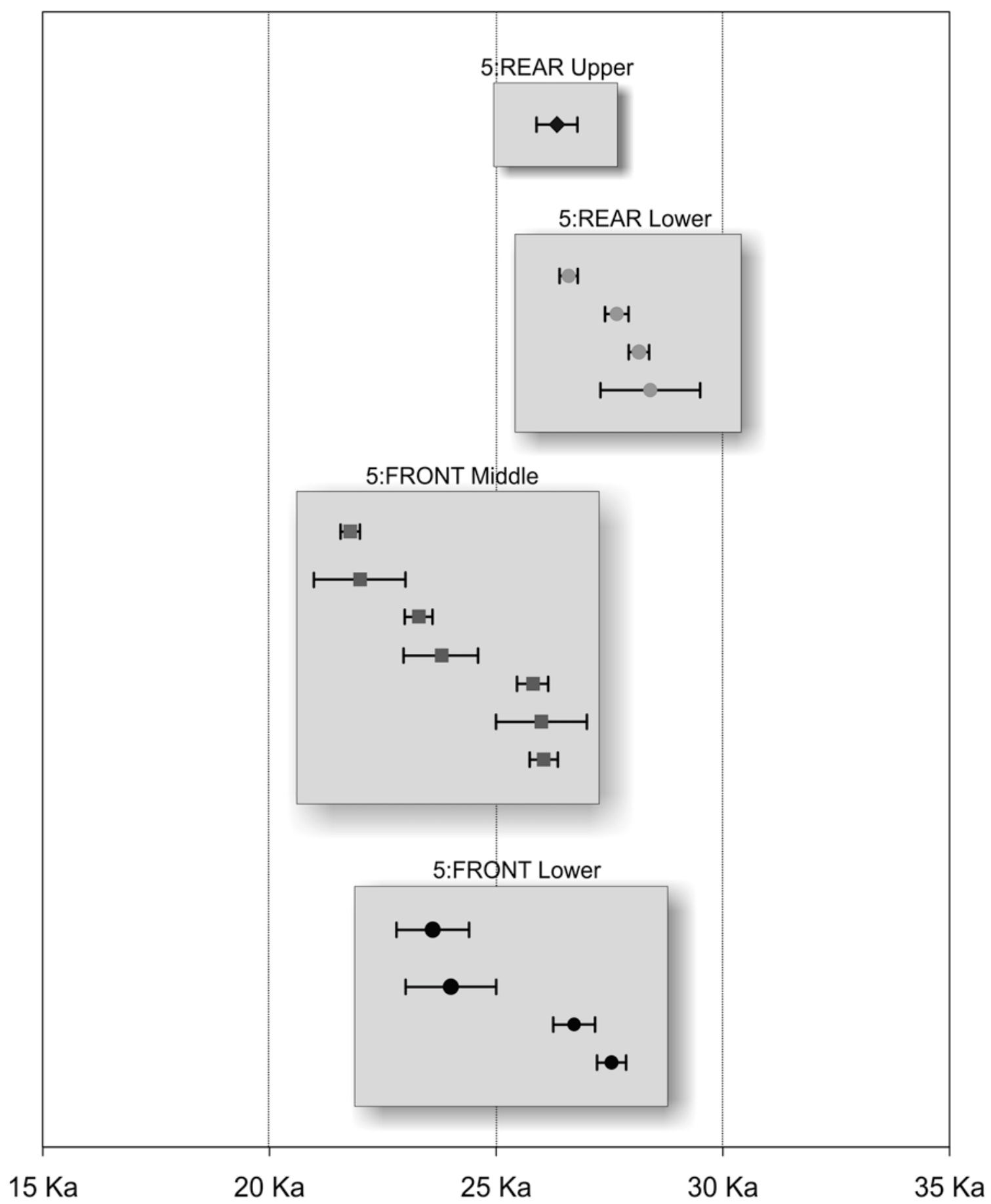

Figure 1 - Datations des niveaux gravettiens de l'Abri Pataud.

Figure 1 - Radiocarbon dates from the Gravettian levels of Abri Pataud. 


\begin{tabular}{|c|c|}
\hline \multicolumn{2}{|c|}{ 5:Front Middle (7 dates) } \\
\hline GrN-4631 & $21780 \pm 215$ \\
\hline Ly-300 & $22000 \pm 1000$ \\
\hline GrN-5009 & $23350 \pm 170$ \\
\hline Ly-100 & $23800 \pm 800$ \\
\hline Gx-1371 & $25815 \pm 330$ \\
\hline OxA-581 & $26000 \pm 1000$ \\
\hline GrN-5012 & $26050 \pm 310$ \\
\hline
\end{tabular}

\begin{tabular}{|c|c|}
\hline \multicolumn{2}{|c|}{ 5:Front Lower (4 dates) } \\
\hline W-151 & $23600 \pm 800$ \\
\hline W-191 & $24000 \pm 1000$ \\
\hline Gx-1369 & $26720 \pm 460$ \\
\hline Gx-1370 & $27545 \pm 320$ \\
\hline
\end{tabular}

\begin{tabular}{|c|c|}
\hline \multicolumn{2}{|c|}{ 5:Rear Upper (1 date) } \\
\hline Gx-1372 & $26340 \pm 450$ \\
\hline
\end{tabular}

\begin{tabular}{|c|c|}
\hline \multicolumn{2}{|c|}{ 5:Rear Lower (4 dates) } \\
\hline GrN-4477 & $26600 \pm 200$ \\
\hline GrN-4662 & $27660 \pm 260$ \\
\hline GrN-4634 & $28150 \pm 225$ \\
\hline OxA-169 & $28400 \pm 1100$ \\
\hline
\end{tabular}

Tableau 1 - Datations des niveaux gravettiens de l'Abri Pataud. Les dates Oxa-581 et Oxa 169 ont été obtenues par procédé AMS.

Table 1 - Radiocarbon dates from the Gravettian levels de Abri Pataud. Oxa-581 et Oxa 169 are AMS dates. échantillons provenant de la partie arrière sont les plus anciens, alors qu'ils devraient être les plus récents, ou tout du moins, beaucoup plus dispersés qu'ils ne le sont. Par contre, les échantillons de la partie avant sont plus récents, et ce, quelque soit la méthode utilisée.

\section{Le niveau 3}

Un autre exemple de problème d'interprétation stratigraphique et culturelle peut être donné à propos du niveau 3 (Gravettien récent), qui conservait sans conteste le plus grand nombre de structures interprétables en terme d'aires d'activités de tous les niveaux gravettiens de l'abri Pataud. Le niveau 3 est caractérisé par une occupation principale, la Lens 2, située en arrière d'une limite naturelle formée par une série de blocs d'effondrements de la voûte parallèlement au fond de l'abri. Cet espace protégé, d'une superficie de $72 \mathrm{~m}^{2}$, semble être l'aire centrale de l'habitat, organisée autour d'une série de foyers alignés, et avait été qualifiée par Hallam L. Movius de « Long House » (Bricker et David 1984 ; Movius 1977). Plusieurs occupations, d'importance inégale, prennent place avant et après cette occupation principale, qui contient $55 \%$ de tout le matériel archéologique inventorié du niveau 3. Les deux premières occupations correspondent au Lens 4 et Lens 3, interstratifiées avec l'Eboulis c et l'Eboulis c'. La dernière occupation correspond à la Lens 1 , séparée de la Lens 2 par l'Eboulis $b$ et surmontée par l'Eboulis a. Les subdivisions en différentes lentilles sont interprétées comme des installations successives, et sont donc les unités interprétatives minimales. La réalisation de remontages lithiques a toutefois montré qu'au sein du débitage récolté par subdivisions stratigraphiques et planimétriques, il était possible de mettre en relation différentes subdivisions du niveau 3. Plus encore, pour l'un des remontages (comportant 27 pièces), quatre des huit subdivisions sont représentées (Nespoulet 1996, 1999, 2000) (tableaux 2 et 3). L'interprétation de la diachronie des occupations au Gravettien récent doit donc être pondérée par ce type d'informations récentes issues du réexamen des collections, même si l'interprétation de la Lens 2 en tant qu'occupation principale ne doit pas, selon nous, être remise en doute.

\section{À quelles séries fait-on référence?}

Les données quantitatives concernant le matériel archéologique gravettien de l'Abri Pataud s'approchent aujourd'hui de l'exhaustivité, par un inventaire commencé en 1958 et poursuivi depuis 1989. Aux décomptes concernant les industries et les faunes déterminables prises en compte par Hallam L. Movius et ses collaborateurs, il convient désormais de rajouter ceux réalisés sur les séries restées inédites après les fouilles.

En prenant en compte le matériel lithique et osseux, on peut avancer l'estimation très vraisemblable ${ }^{3}$ de 1071444 pièces au total, dont 255069 pièces lithiques (23,8 \%) et 816375 pièces fauniques (76,2\%). Le niveau 5 est le plus riche (508 872 pièces estimées, soit 47,5\%), suivi du

(3) L'estimation se base sur un décompte précis et exhaustif de tous les sacs de "débitage ». Leur volume a été pris en compte. 


\begin{tabular}{|c|c|c|c|c|c|c|}
\hline & Etape & Ordre & $N^{\circ}$ & Zone & Subdivision & Type \\
\hline \multirow{16}{*}{$\begin{array}{l}\bar{U} \\
\text { w } \\
\frac{1}{1} \\
\frac{1}{2}\end{array}$} & \multirow{3}{*}{1} & $A$ & 23420 & GV & lens $2 \mathrm{~b}$ & Eclat \\
\hline & & \multirow{2}{*}{ B } & 22048 & $\mathrm{FI}$ & eboulis b & \multirow{2}{*}{ Eclat } \\
\hline & & & 23227 & $\mathrm{FI}$ & lens 2 & \\
\hline & \multirow{4}{*}{2} & \multirow{3}{*}{1} & 22705 & $\mathrm{FI}$ & lens 1 & \\
\hline & & & 22116 & $\mathrm{FI}$ & eboulis b & Lame encochée \\
\hline & & & 22065 & $\mathrm{FI}$ & eboulis b & \\
\hline & & 2 & 21992 & $\mathrm{FI}$ & lens 2 a & Lame utilisée \\
\hline & \multirow{2}{*}{3} & 3 & 22366 & $\mathrm{El}$ & lens 2 & Eclat \\
\hline & & 4 & 22410 & FII & lens 2 & Eclat \\
\hline & \multirow{3}{*}{4} & 5 & 22885 & FIII & lens 2 & Lame utilisée \\
\hline & & \multirow{2}{*}{6} & 21519 & $\mathrm{FI}$ & lens 2 & \multirow{2}{*}{ Lame utilisée } \\
\hline & & & 22444 & $\mathrm{FI}$ & lens 2 & \\
\hline & 5 & 7 & 22877 & FII & lens 1 & Eclat \\
\hline & \multirow{3}{*}{6} & \multirow{3}{*}{8} & 22361 & $\mathrm{EI}$ & lens 2 & \multirow{3}{*}{ Lame utilisée } \\
\hline & & & 22311 & $\mathrm{GI}$ & lens 2 & \\
\hline & & & 28230 & GVI & eboulis c & \\
\hline \multirow{6}{*}{$\begin{array}{l}\text { U } \\
\text { के } \\
\frac{1}{\alpha} \\
\frac{1}{2}\end{array}$} & \multirow{4}{*}{7} & \multirow{2}{*}{9} & 927 & FV & eboulis $b$ & \multirow{2}{*}{ Microgravette } \\
\hline & & & 1919 & GII & lens 2 & \\
\hline & & \multirow{2}{*}{10} & 25640 & $\mathrm{El}$ & lens 2 & \multirow{2}{*}{ Lamelle } \\
\hline & & & 22450 & $\mathrm{FI}$ & lens 2 & \\
\hline & 8 & \multirow{2}{*}{11} & 27502 & FII & lens $2 a$ & \multirow{2}{*}{ Eclat } \\
\hline & 9 & & 23133 & $\mathrm{FI}$ & general & \\
\hline \multirow{5}{*}{$\begin{array}{l}m \\
w \\
\text { w } \\
\frac{1}{1}\end{array}$} & \multirow{4}{*}{10} & 12 & 23035 & $\mathrm{FI}$ & general & lamelle \\
\hline & & 13 & 23321 & $\mathrm{FI}$ & general & Eclat \\
\hline & & 14 & 25611 & $\mathrm{El}$ & lens 2 & Eclat \\
\hline & & 15 & 1048 & El & lens 2 main & Lamelle \\
\hline & 11 & 16 & 958 & $\mathrm{FI}$ & lens 1 & Nucléus \\
\hline
\end{tabular}

Tableau 2 - Niveau 3. Pièces du remontage $n^{\circ} 1$ dans leur ordre de débitage.

Table 2 - Level 3. Pieces of the refitting $n^{\circ} 1$ in their debitage order.

Tableau 3 - Niveau 3.

Remontage $n^{\circ} 1$. Récapitulatif des différentes subdivisions présentes dans le remontage.

Table 3 - Level 3.

Refitting $n^{\circ}$ : different subdivisions represented.

\begin{tabular}{|c|c|}
\hline $\begin{array}{c}\text { Subdivision } \\
\text { de la couche 3 }\end{array}$ & $\begin{array}{c}\text { nombre } \\
\text { de pièces }\end{array}$ \\
\hline Lens 1 & 3 \\
\hline Eboulis b & 4 \\
\hline Lens 2 & 16 \\
\hline Eboulis c & 1 \\
\hline Général & 3 \\
\hline Total & $\mathbf{2 7}$ \\
\hline
\end{tabular}

niveau 4 (341 906 pièces, soit 31,9\%), du niveau 3 (126 847 pièces, soit $11,8 \%$ ) et du niveau 2 (93 819 pièces, soit 8,4 \%) (tabl. 4).

L'outillage lithique représente 18134 pièces décomptées à ce jour, soit $7,1 \%$ de la totalité de l'industrie lithique du Gravettien. C'est le niveau 4 qui présente le plus grand taux de transformation des supports $(8,8 \%$ de l'industrie lithique), suivi des niveaux 2 (7,3\%), 5 (6\%) et $3(4,9 \%)$ (tabl. 5). La variabilité de ce taux transformation, dont l'analyse et l'interprétation dépasse le cadre de cette présentation, a des causes multiples : représentativité de la zone fouillée (donc de l'échantillon), de la fonction du site, de l'utilisation des supports non retouchés et, bien sûr, de la stratégie d'approvisionnement en matière première. Dans ce dernier cas, la quantité des silex du Bergeracois (ou leur « équivalent ", comme dans le niveau 4 (Pottier 2005) et des autres silex importés influe directement sur les taux de transformation. De récentes études détaillées des matières premières ont été réalisées (Chiotti et al. 2003, 2004), certaines sont en cours (Léoz 2001; Pottier 2005) et nous n'aborderons ici que quelques aspects limités. La très grande quantité de matériel archéologique à prendre en compte, si l'on veut tendre à l'exhaustivité de l'étude, est le principal frein aux études sur les matières premières des séries gravettiennes de l'Abri Pataud. Elles ont toutefois été réalisées pour les niveaux 2, 3 et 4 et sont en cours pour le niveau 5.

Depuis quelques années, sur la base de certaines observations réalisées grâce à la lithothèque régionale du Musée national de Préhistoire ${ }^{4}$, il s'est avéré que plusieurs types de matériaux (provenant de Charente ou des Landes par exemple) restaient probablement non encore clairement identifiés.

Nous manquons de certaines données pour le niveau 5 (Gravettien ancien) pour l'instant. On peut toutefois faire remarquer la grande diversité des matériaux et l'importance du silex du Bergeracois pour la production des pointes de La Gravette.

Le niveau 4 (Gravettien moyen) se caractérise par la présence des silex blancs patinés avec une utilisation préférentielle pour le façonnage des burins du Raysse et des pièces esquillées.

La niveau 3 (Gravettien récent), avec $11 \%$ de silex importé, est marqué par l'utilisation préférentielle du silex du Bergeracois pour les supports de burins (en particulier les burins sur troncature) : près du tiers sont réalisés dans cette matière.

Le niveau 2 (Gravettien final) est marqué par l'introduction de grandes lames déjà débitées en silex du Bergeracois. Dans ce niveau $16 \%$ du silex est importé. 


\begin{tabular}{|c|c|c|c|c|c|}
\hline & Lithique & Faune & Total & Volume fouillé & Surface fouillée \\
\hline Couche 2 & 25405 & 68414 & 93819 & $20 \mathrm{~m} 3$ & $120 \mathrm{~m} 2(196 \mathrm{~m} 2)^{*}$ \\
\hline Couche 3 & 33786 & 93061 & 126847 & $34 \mathrm{~m} 3$ & $120 \mathrm{~m} 2(168 \mathrm{~m} 2)^{\star}$ \\
\hline Couche 4 & 99878 & 242028 & 341906 & $30 \mathrm{m3}$ & $91 \mathrm{~m} 2$ \\
\hline Couche 5 & 96000 & 412872 & 508872 & $28 \mathrm{~m} 3$ & $84 \mathrm{~m} 2$ \\
\hline Total & $\mathbf{2 5 5 0 6 9}$ & $\mathbf{8 1 6} \mathbf{3 7 5}$ & $\mathbf{1 0 7 1 ~ 4 4 4}$ & $\mathbf{1 1 0 ~ \mathrm { m } 3}$ & - \\
\hline
\end{tabular}

Tableau 4 - Décompte du matériel archéologique du Gravettien de l'Abri Pataud. Les chiffres en italique correspondent à des estimations.

*Les surfaces fouillées étaient plus grandes que les surfaces décapées contenant du Matériel archéologique.

Table 4 - Count of the Gravettian archaeological assemblage of Abri Pataud. Italic numbers are estimated. ${ }^{*}$ Excavated surfaces were larger than the surfaces with archaeological artefacts.

\begin{tabular}{|c|c|c|c|}
\hline Niveau & Lithique & \multicolumn{2}{|c|}{ outillage } \\
\hline Couche 2 & 25405 & 1845 & $7,3 \%$ \\
\hline Couche 3 & 33786 & 1656 & $4,9 \%$ \\
\hline Couche 4 & 99878 & 8833 & $8,8 \%$ \\
\hline Couche 5 & 96000 & 5800 & $6 \%$ \\
\hline
\end{tabular}

Tableau 5 - Décomptes de la totalité de l'industrie lithique des niveaux gravettiens de l'Abri Pataud.

Table 5 - Count of the totality of the Gravettian flint industry of the Abri Pataud.

\section{L'évolution typologique du Gravettien : quelques tendances}

De nombreuses études typologiques ont été réalisées sur les séries gravettiennes de l'abri Pataud, et nous limiterons ici notre présentation aux fréquences de 6 grandes catégories typologiques : grattoirs, burins dièdres, burins sur troncature, pointe de La Gravette, microgravettes et lamelles à dos. Elles sont basées sur les derniers décomptes disponibles, qui prennent en compte l'outillage découvert dans les sacs de « débitage ».

La parenté typologique entre les niveaux 5 et 3 est toujours d'actualité. Elle s'exprime par des tendances similaires. En particulier, il faut noter la présence, dans ces deux industries, d'une proportion importante de grattoirs. Dans le niveau 5 , certains sont caractéristiques : robustes, façonnés sur éclats larges et épais, très souvent corticaux, ils ont été qualifiés de « coupoirs » par H. M. Bricker (Bricker 1973, 1995). Différents, mais eux aussi souvent façonnés sur des éclats corticaux, les grattoirs sur " éclats informes " sont caractéristiques du niveau 3. Mais le point commun le plus évident reste la présence en abondance de pointes de La Gravette et de microgravettes dans les deux industries.

\section{Des burins variés}

Sous des appellations typologiques similaires, les formes de burins présentent des différences importantes entre les

différentes industries. Le rôle de nucléus a été démontré pour les burins du Raysse du niveau 4 (Pottier 2005) et argumenté pour le niveau 3. Le statut particulier du burin de Noailles n'est plus à démontrer, et à l'abri Pataud, sa variabilité, comme sa fonction, demeurent un sujet de discussions. Enfin les burins dièdres présentent des différences importantes, en particulier concernant leur type de supports, entre les niveaux 5, 4 et 2.

Des lamelles dans tous les niveaux

C'est probablement à travers la production et le façonnage des lamelles que les plus grandes différences peuvent s'observer. Bien que de nombreuses questions restent encore posées, les études récentes et en cours apportent des éléments de réponse. La présence de lamelles de la Picardie dans le niveau 4 est maintenant établie. D'une façon générale, chaque industrie gravettienne à l'Abri Pataud exprime une façon de faire différente. L'originalité de chaque production lamellaire apparaît ainsi comme l'un des sujets les plus prometteurs pour la caractérisation de ces industries.

\section{Perspectives}

Nous n'avons pas présenté ici les données archéozoologiques, en grande partie inédites, alors qu'elles sont indispensables pour caractériser la fonction du site, donc pour orienter l'interprétation des assemblages lithiques. Une 
synthèse récente sur les faunes du Gravettien (Vannoorenberghe 2004) permettra, nous espérons dans un futur proche, de présenter ces résultats.

La révision de la séquence stratigraphique, en particulier d'un point de vue géoarchéologique, semble aujourd'hui indispensable pour mieux appréhender ce gisement complexe. II a été initié en 2004 , et devrait se poursuivre en 2005, en même temps qu'une fouille limitée au niveau 2. Les collections de l'Abri Pataud présentent encore un potentiel important de nouvelles études. II reste encore beaucoup à faire pour mieux caractériser la technologie lithique du gravettien, pour corréler les données des industries avec celles de la faune et des autres productions, comme la parure et l'art.

\section{BIBLIOGRAPHIE}

BRICKER H. M. 1973 - The Perigordian IV and Related Cultures in France, Department of Anthropology, Harvard University, Ph. D. Dissertation, 1849 p.

BRICKER H. M., DAVID N. 1984 - Excavation of the Abri Pataud, les Eyzies (Dordogne), The Perigordian VI (Level 3) assemblage, Bulletin of the American School of Prehistoric Research, Peabody Museum, Harvard University, $n^{\circ} 34,109 p$.

BRICKER H. M. et MELLARS P. A. 1987 - Datation C14 de l'abri Pataud (Les Eyzies, Dordogne) par le procédé "Accélérateur-Spectromètre de masse», L'Anthropologie, tome $91, \mathrm{n}^{\circ} 1$, p.227-234.

BRICKER H. M. (sous la direction de) 1995 - Le Paléolithique supérieur de l'abri Pataud, Dordogne, les fouilles de H..L. Movius Jr, Maison des Sciences de l'Homme, Documents d'Archéologie Française, Paris, $n^{\circ} 50$, $328 \mathrm{p}$.

BROOKS A. S. 1979 - The significance of variability in Paleolithic assemblages : An Aurignacian example from southwestern France, thesis of Department of Anthropology, Harvard University, Cambridge, Massachussetts, 1057 p.

BROOKS A. S. 1995 - L'Aurignacien de l'abri Pataud, niveaux 6 à 14, in : Bricker, Le Paléolithique supérieur de l'Abri Pataud (Dordogne) : les fouilles de H. L. Movius Jr., Documents d'Archéologie Française, Editions de la maison des sciences de l'homme, Paris, p. 167-222.

CHIOTTI L. 1999 - Les industries lithiques des niveaux aurignaciens de l'Abri Pataud, Les Eyzies de Tayac (Dordogne) : étude technologique et typologique. Thèse de Doctorat. Muséum national d'Histoire naturelle, Paris, 2 tomes, $839 \mathrm{p}$.

CHIOTTI L., LEOZ L. E., NESPOULET R. et POTTIER C. 2003 - Quelques exemples de stratégies d'approvisionnement dans l'Aurignacien et le Gravettien à l'abri Pataud (Dordogne), In : Actes de la Table ronde internationale,
Aurillac, 20-22 juin 2002, Préhistoire du Sud-Ouest, supplément $\mathrm{n}$ 05, pp. 115-122.

CHIOTTI L., NESPOULET R. et POTTIER C., 2004 Contribution des collections du Paléolithique supérieur de l'abri Pataud (Les Eyzies-de-Tayac, Dordogne, France) à la connaissance de la gestion des matières premières siliceuses dans le nord de l'Aquitaine, Actes du XIV ${ }^{e}$ Congrès de I'U.I.S.P.P., Section 6 : Le Paléolithique supérieur, 2-8 septembre 2001, Oxford, BAR International Series, $\mathrm{n}^{\circ}$ 1240, 2004, pp. 73-82.

LEOZ L. E. 2001 - Contribution à l'étude du Gravettien de l'abri Pataud (Dordogne) : étude techno-typologique de l'industrie du niveau 5 : Front, attribuée au Périgordien IV. Bulletin de la Société Historique et Archéologique du Périgord, CXXVIII, pp. 579-596.

MOVIUS H. L. Jr. 1971 - Radiocarbon dating of the Upper Palaeolithic sequence at the Abri Pataud, Les Eyzies (Dordogne), The Origin of Homo sapiens (Ecology and Conservation, 3) UNESCO, p. 253-260.

MOVIUS H. L. Jr. 1975 - Excavation of the abri Pataud, Les Eyzies (Dordogne): Contributors, American School of Prehistoric Research, Peabody Museum, Harvard University, Cambridge, Massachusetts, Bulletin $n^{\circ} 30$, $305 \mathrm{p}$.

MOVIUS H. L. Jr. 1977 - Excavation of the abri Pataud, Les Eyzies (Dordogne): Stratigraphy, American School of Prehistoric Research, Peabody Museum, Harvard University, Cambridge, Massachusetts, Bulletin $n^{\circ} 31,167$ p.

NESPOULET R. 1996 - Le Périgordien VI de l'abri Pataud, Les Eyzies-de-Tayac, Dordogne. Etude technologique et typologique de l'industrie lithique de la couche 3 , thèse de doctorat, Muséum National d'Histoire Naturelle, Paris, 260 p.

NESPOULET R. 1999 - Remontage d'une microgravette dans une séquence de débitage laminaire du Gravettien final de l'Abri Pataud (Les Eyzies-de-Tayac, Dordogne). Niveau 3 : Périgordien VI, Préhistoire du Sud-Ouest, $\mathrm{n}^{\circ} 6$, pp. $57-77$.

NESPOULET R. 2000 - Le Gravettien final de l'abri Pataud, Les Eyzies-de-Tayac, Dordogne. Nouvelles données technologiques et typologiques sur l'industrie lithique provenant du niveau 3, L'Anthropologie, vol. 104, n 1, 2000, pp. 63-120.

POTTIER C. 2005 - Le Gravettien moyen de l'abri Pataud (Dordogne, France) : le niveau 4 et l'éboulis 3/4. Etude technologique et typologique de l'industrie lithique, Thèse de doctorat, Muséum national d'Histoire naturelle, Paris, $393 \mathrm{p}$.

VANNOORENBERGHE A. 2004 - Contribution à la connaissance des comportements de subsistance des gravettiens dans le Sud-Ouest de la France. La Faune du 
Gravettien ancien de l'abri Pataud (Les Eyzies-de-Tayac, Dordogne). Thèse de doctorat, Muséum national d'Histoire naturelle Paris, $430 \mathrm{p}$.
VOGEL J. C., WATERBOLK H. T. 1994 - Chronologie du peuplement gravettien sur les côtes de Méditerranée occidentale, XXIVe Congrès préhistorique de France, Carcassonne, 26-30 septembre 1994, Les faciès leptolithiques du nord-ouest méditerranéen : milieux naturels et culturels, pp. 127-138. 